\title{
Security Analysis of Machine Learning-Based PUF Enrollment Protocols: A Review
}

\author{
Sameh Khalfaoui ${ }^{1,2}, * \mathbb{D}$, Jean Leneutre ${ }^{2}$, Arthur Villard ${ }^{1}$, Ivan Gazeau ${ }^{1}$, Jingxuan Ma ${ }^{1}$ and Pascal Urien ${ }^{2}$ \\ 1 EDF R\&D, 7 Boulevard Gaspard Monge, 91120 Palaiseau, France; arthur.villard@edf.fr (A.V.); \\ ivan.gazeau@edf.fr (I.G.); jingxuan.ma@edf.fr (J.M.) \\ 2 LTCI, Télécom Paris, Institut Polytechnique de Paris, 19 Place Marguerite Perey, 91120 Palaiseau, France; \\ jean.leneutre@telecom-paris.fr (J.L.); pascal.urien@telecom-paris.fr (P.U.) \\ * Correspondence: sameh.khalfaoui@edf.fr; Tel.: +33-633132159
}

Citation: Khalfaoui, S.; Leneutre, J.; Villard, A.; Gazeau, I.; Ma, J.; Urien, P. Security Analysis of Machine Learning-Based PUF Enrollment Protocols: A Review. Sensors 2021, 21, 8415. https://doi.org/10.3390/ s21248415

Academic Editors: Tiago Cruz and Paulo Simões

Received: 29 October 2021

Accepted: 13 December 2021

Published: 16 December 2021

Publisher's Note: MDPI stays neutral with regard to jurisdictional claims in published maps and institutional affiliations.

Copyright: (c) 2021 by the authors. Licensee MDPI, Basel, Switzerland. This article is an open access article distributed under the terms and conditions of the Creative Commons Attribution (CC BY) license (https:/ / creativecommons.org/licenses/by/ $4.0 /)$.

\begin{abstract}
The demand for Internet of Things services is increasing exponentially, and consequently a large number of devices are being deployed. To efficiently authenticate these objects, the use of physical unclonable functions (PUFs) has been introduced as a promising solution for the resourceconstrained nature of these devices. The use of machine learning PUF models has been recently proposed to authenticate the IoT objects while reducing the storage space requirement for each device. Nonetheless, the use of a mathematically clonable PUFs requires careful design of the enrollment process. Furthermore, the secrecy of the machine learning models used for PUFs and the scenario of leakage of sensitive information to an adversary due to an insider threat within the organization have not been discussed. In this paper, we review the state-of-the-art model-based PUF enrollment protocols. We identity two architectures of enrollment protocols based on the participating entities and the building blocks that are relevant to the security of the authentication procedure. In addition, we discuss their respective weaknesses with respect to insider and outsider threats. Our work serves as a comprehensive overview of the ML PUF-based methods and provides design guidelines for future enrollment protocol designers.
\end{abstract}

Keywords: Internet of Things; authentication; physical unclonable function; machine learning

\section{Introduction}

The deployment of smart sensors is exponentially increasing to cover consumer oriented services and the requirements of industrial scenarios [1]. The high popularity of Internet of Things (IoT) products is pressuring manufacturers to opt for rush to market behavior in order to comply with their clients' needs. Thus, they tend to overlook the importance of ensuring the security of these resource-constrained devices, which might create a potential attack vector once they are deployed. Moreover, the National Institute of Standards and Technology (NIST) has recently introduced new regulations, NISTIR 8259A [2], for the United States IoT market regarding the security of new devices and the data collection. On the other hand, the European Telecommunications Standards Institute (ETSI) has released similar cybersecurity guidelines, in the ETSI EN 303,645 report [3], for the IoT consumer market in Europe and in the United Kingdom. Manufacturers need more comprehensive and easy-to-adopt security solutions in order to keep pace with the regulations. Therefore, the application of an entity authentication procedure that is suitable for the IoT context is crucial. By doing so, we eliminate the risk related to allowing a malicious object into the network of a user. This secure enrollment process ensures that the communicating IoT nodes are trustworthy.

Numerous entity authentication solutions have been proposed to verify the identity and the origin of the IoT object in question. The identity-based [4] and certificate-based techniques [5] represent promising candidates when combined with a lightweight cryptographic algorithm that is supported by a resource-constrained device. However, we 
encourage the avoidance of exploiting any pre-established security knowledge between the verifier and the prover to facilitate the integration of our solution with a secure device pairing scheme [6,7]. The no prior secrets condition is motivated by the unfeasibility of managing the scalability issues of public key infrastructure due to the growing numbers of heterogeneous IoT devices. A third possible alternative is to exploit a One-Time Password (OTP) system [8] that authenticates the object using a trusted third party. Nevertheless, this technique requires the IoT device to communicate independently with a remote OTP server. As a consequence, we would prefer to perform the authentication process prior to associating the IoT object with the network of the user. Therefore, the OTP solution would not be compliant with our requirements. A final alternative is to use of a hardwarebased enrollment protocol that relies on a secure element such as a PUF [9] onboard the object. This method provides a lightweight and a cost-effective authentication system that is adequate with the IoT context. Several integrated circuit vendors have opted for a hardware-level technology approach for securing the use of the IoT object through physical unclonable functions. These secure hardware elements serve multiple objectives, such as device identification, secure key management and secure boot functionality. This technology has been applied to IoT products, but it can also play a major role in the security systems used in other industrial areas, such as the vehicular context, as discussed in [9]. This role can cover, for example, the vehicle component identification [10] or cryptographic key management for securing a vehicular ad hoc network [9].

Unfortunately, a growing number of the recently proposed PUFs, such as the InterposePUF [11] and the Double Arbiter PUF (DAPUF) [12], have been proven vulnerable against a variety of machine learning $(\mathrm{ML})$ attacks that aim at modeling their behavior by collecting a sufficient number of challenge-response pairs (CRP) [13,14]. Therefore, several enrollment protocols have intentionally exploited some vulnerable PUF architectures to create ML models that simulate their behavior [15,16]. The work of Pour et al. [17] briefly discusses the benefits of exploiting these modeling methods in an industrial scenario. These advantages include reducing the time that is required to enroll a large number of devices and the storage space that should be used to store the challenge-response pairs. As a consequence, a server can efficiently many IoT devices. The existing reviews of PUF-based enrollment procedures tend to focus on the traditional use of these hardware circuits through the storage of the CRPs [18,19]. Other reviews concentrate on reviewing the vulnerabilities of these PUF architectures to ML modeling attacks [20-22]. However, we have noticed that they overlook the exploitation of these ML modeling techniques in order to reduce the required storage space while maintaining the same level of security.

In this paper, we provide an in depth overview of the state-of-the-art model-based PUF enrollment protocols. We classify the existing proposals based on two architectures. In addition, we describe the different components of the protocols and we discuss their respective weaknesses. Additionally, we evaluate the robustness of the identified enrollment protocols against an insider threat scenario that targets the secrecy of the given PUF ML model. Our paper serves as a comprehensive overview of the scalable PUF-based methods that have been used so far to perform the enrollment procedures of IoT objects.

The rest of the paper is organized as follows. Section 2 introduces the basics of the PUF technology and the different machine learning techniques that are applied to model these circuits. Section 3 presents the two enrollment architectures and details their building blocks. Section 4 provides an extensive study of the existing model-based PUF enrollment protocols in light of the proposed architectures. Section 5 discusses the advantages and the limitations of these architectures, alongside of the weaknesses identified in the described schemes. Furthermore, it outlines the impacts of the insider attack scenario on the security of the authentication process and provides future research directions to mitigate the threats. Lastly, Section 6 concludes the work. 


\section{Preliminaries}

In this section, we provide background on the physical unclonable functions, and we describe their most commonly used constructions for authenticating physical systems. Furthermore, we introduce a selection of the most effective machine learning techniques for modeling the behavior of PUF circuits.

\subsection{Physical Unclonable Function}

A physical unclonable function is a secure element that identifies, in a unique manner, a specific device through a challenge-response process. This paired information represents the pattern of responses when we have a set of specific challenges as inputs. This function has to be unclonable and unique for each device, since it relies on physical randomness that can be either explicitly introduced or intrinsically present in the physical system [23]. The micro-variations in the hardware system allow the same construction of a PUF to provide unique responses when deployed on different circuits. Thus, these variations play the role of the seed in a random response generator.

There are two major categories of PUFs based on the source of the randomness. The first category of PUFs, referred to as electronic PUFs, rely on a number of micro-physical parameters that are hidden from the physical observation inside the electronic circuit. These parameters can be detected only when they are needed to produce the unique responses. These variables include the time, the frequency, the current or the voltage, the bistable states and the capacitance [24]. The second category of PUFs, referred to as non-electronic PUFs, includes the PUF elements that rely on unique characteristics of the physical system in a non-electronic manner, such as the use of light in optical PUFs [25] and the radio variations in the RF-PUF [26]. Readers that are eager to learn more about the different PUF architectures can consult the review in [27].

The electronic PUF elements can be further classified into two categories: strong PUFs and weak PUFs. Strong PUFs provide many challenge-response pairs, which makes them suitable for the authentication operations. This is explained by the possibility of conducting numerous authentication attempts using different CRPs in each session without the need to reuse the same credentials. Thus, they represent interesting candidate solutions in the context of multi-user IoT objects. Weak PUFs provide fewer CRPs. However, these PUFs have been increasingly popular as internal key generators [28,29]. In this work, we focus on the authentication protocols that are based on strong PUFs.

\subsubsection{Arbiter PUF}

The Arbiter PUF [30] is one of the most popular electronic PUFs that are exploited for authentication operations. This PUF's architecture is based on a comparison of the travel times of two electrical signals propagating down two symmetrical paths. The uniqueness of the responses is based on the manufacturing variations in the creation of these two paths. This PUF is constructed using a pre-determined number of 22 cells that connect these paths. The choice of connection routes depends entirely on the $l$ challenge bits $C[x], x \in[1, l]$. Finally, the arbiter component decides which signals has arrived first, and accordingly outputs the associated binary response, as illustrated in Figure 1.

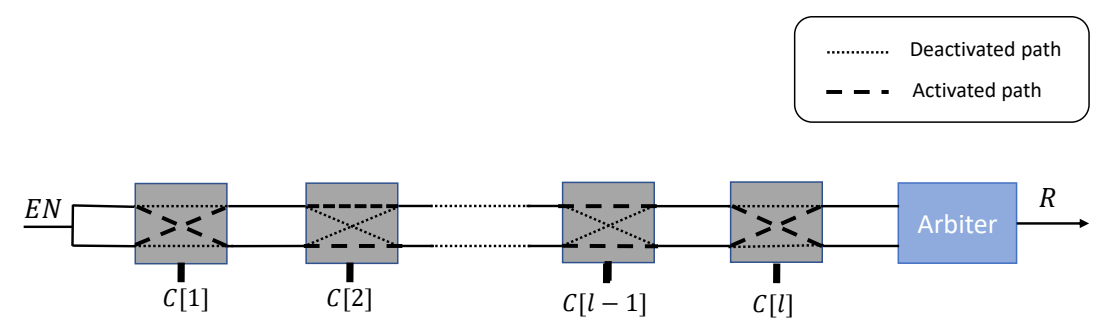

Figure 1. Arbiter PUF architecture. 


\subsubsection{XOR Arbiter PUF}

This PUF architecture is a variant of the previously described Arbiter PUF. It has been developed as way to enhance the complexity of the mapping function between the input challenges and the output responses. As highlighted in Figure 2, this construction uses $n$ independent Arbiter PUFs, and it applies an XOR operation on their individual responses to obtain the output response $R$. However, the stability of the responses is highly affected by the number $n$ of applied Arbiter PUFs.

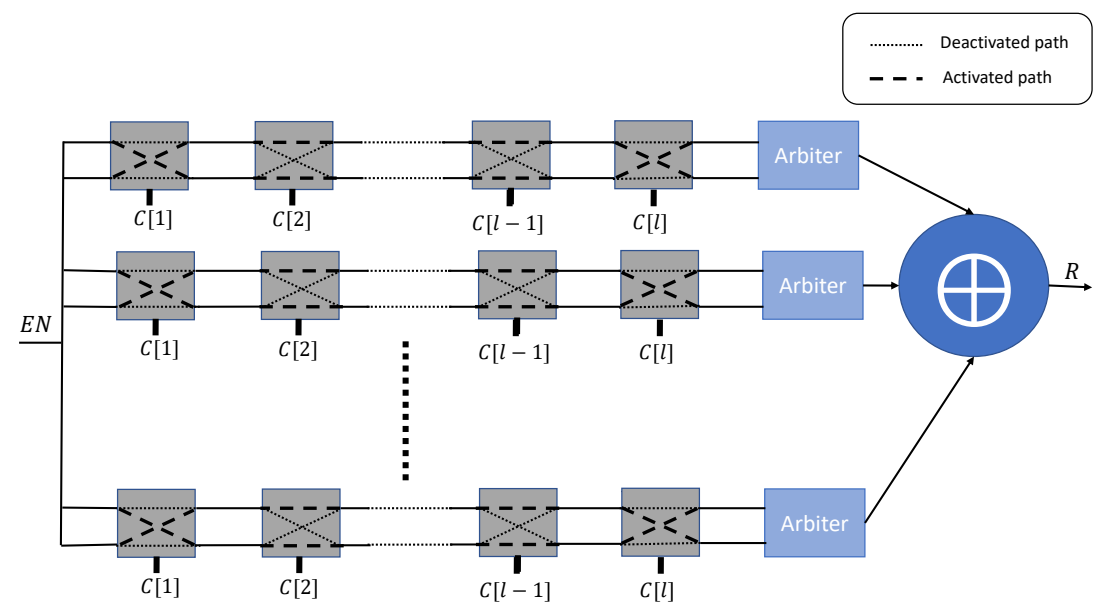

Figure 2. $n$-XOR Arbiter PUF architecture.

Yu et al. [31] has presented another variant of the XOR Arbiter PUF by applying a different challenge at each stage. $n$ challenges can be constructed by applying a linearfeedback shift register (LFSR) to the received root challenge $C$, as shown in Figure 3.

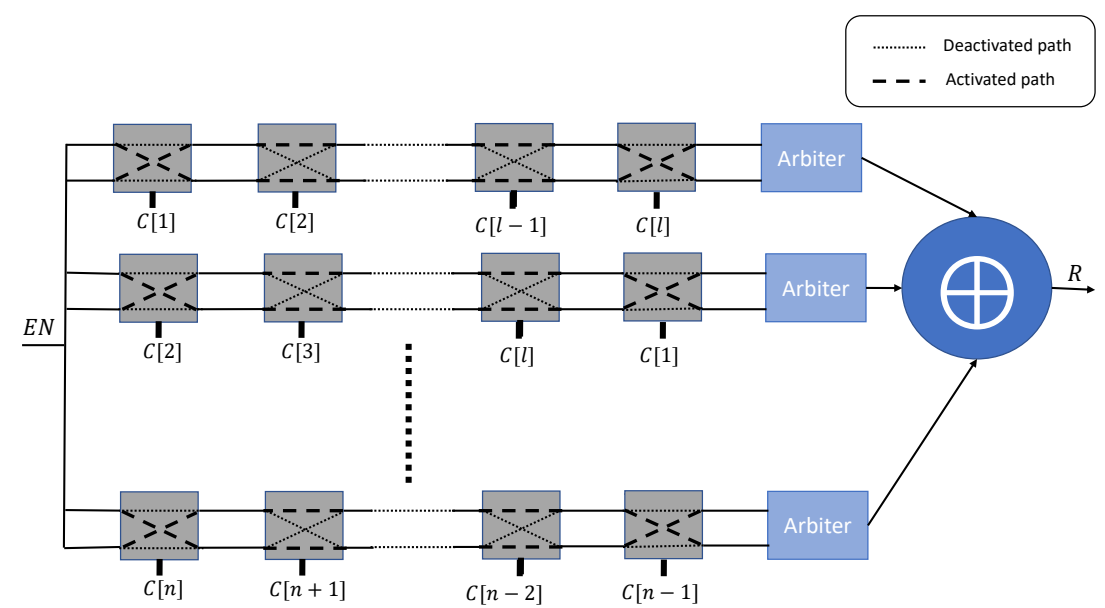

Figure 3. $n$-XOR Arbiter PUF variant with a derivative challenge for each stage.

\subsubsection{Logically Reconfigurable PUF}

The Logically Reconfigurable PUF (LR-PUF) [32] represents a secure hardware element that has the ability to change its challenge-response behavior. The reconfigurability aspects can be achieved in the context of integrated circuits through the use of a field-programmable gate array (FPGA). These PUF circuits should guarantee two properties: forward and backward-unpredictability. The former property assures that the challenge-response pairs collected before the reconfiguration is invalid. Thus, the adversary cannot model the current PUF behavior through the use of previously collected CRPs. The latter property guarantees that an adversary with access to the current reconfigured PUF cannot estimates the responses before the reconfiguration. The work of Liu et al. [33] has identified two type of LR-PUFs: circuit-based reconfigurable PUFs (C-RPUFs) and algorithm-based reconfigurable 
PUFs (A-RPUFs). The former category uses reconfigurable components onboard the circuit to change the original construction. Thus, this hardware level modification changes the behavior of the PUF. The latter category keeps the original hardware components, and instead, it applies a configurable algorithm to change the mapping between the challenges and the responses.

\subsection{Modeling of PUF Designs}

\subsubsection{Logistic Regression}

Logistic regression (LR) is a well-known supervised learning technique. This method models the probability of a discrete outcome that is associated with a specific input variable. The LR learning algorithm is based on the sigmoid function and a set of weights that are learned by using the training dataset. The logistic regression technique is commonly used for the binary classification problems. Therefore, this methodology has been applied, in $[14,21,22]$, to model the behavior of a binary output PUF such as the Arbiter PUF variants, described in Sections 2.1.1 and 2.1.2.

The resilient propagation (RProp) [34] has been an increasingly popular algorithm to optimize the weight coefficients of the logistic regression technique. This is due to its ability to dynamically adapt the step size, independently, for each weight. This technique has been applied in the work of Rührmair et al. $[13,35]$ to model the $x$-XOR Arbiter PUF with $x \leq 5$ and with an accuracy of up to $98 \%$. Furthermore, the work of Khalafalla and Gebotys [22] has exploited a LR learning technique with a linear decision boundary against a more complex Arbiter PUF variant (DAPUF [12]). This method has yielded an enhanced modeling accuracy of up to $99 \%$ with less challenge-response pairs and with cheaper computing resources.

\subsubsection{Support Vector Machine}

The support vector machine (SVM) algorithm [36] has been widely used in classification tasks. The objective of this technique is to find an optimal hyperplane in a $\mathrm{N}$ dimentional space that separates the data points. This hyperplane should classify the data points in a way that maximizes the distance between the identified classes. The Figure 4 illustrates a binary classification problem where the optimal hyperplane is represented as a continuous line. However, the dashed lines represent the other candidate hyperplanes that do not provide the maximum margin between the two classes. Due to the popularity of the SVM algorithm in the binary classification tasks, it has been used in numerous studies $[13,22,35,37,38]$ to model some variants of the Arbiter PUF with limited complexity.

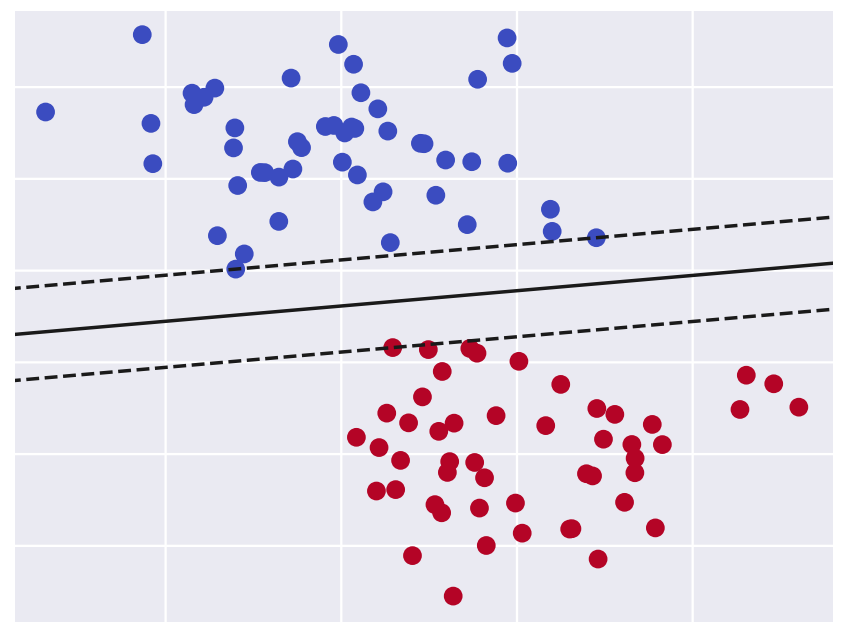

Figure 4. Binary classification problem using the support vector machine algorithm. 


\subsubsection{Artificial Neural Networks}

The artificial neural network (ANN) [39] is a system that imitates the function of the human brain through the use of multiple artificial neurons. This system consists of a number of neuron layers that are referred to as an input layer, one or multiple hidden layers and the classifier layer, as illustrated in Figure 5. Each neuron in the network is connected to another and has an associated weight and a threshold. These parameters are updated over time based on the training data to improve the prediction accuracy of the neural network model.

The ANNs that consist of single hidden layers are referred to as single layer perceptrons (SLPs) and they are only applicable in the case of linearly separable data. Therefore, multiple layer perceptrons (MLPs) are used in the case of non-linear problems. In the context of PUF modeling, a great body of work exploits the power of these model to either attack the state-of-the-art PUF constructions or to demonstrate their resiliency against ML modeling attempts. Unfortunately, a growing number of the proposed ML-resistant PUFs, such as the Interpose-PUF [11] and the 9-Xor Arbiter PUF [40], have been proven vulnerable against a variety of ANN attacks that aim at modeling their behavior by accessing a sufficient number of challenge-response pairs [14,41-43].

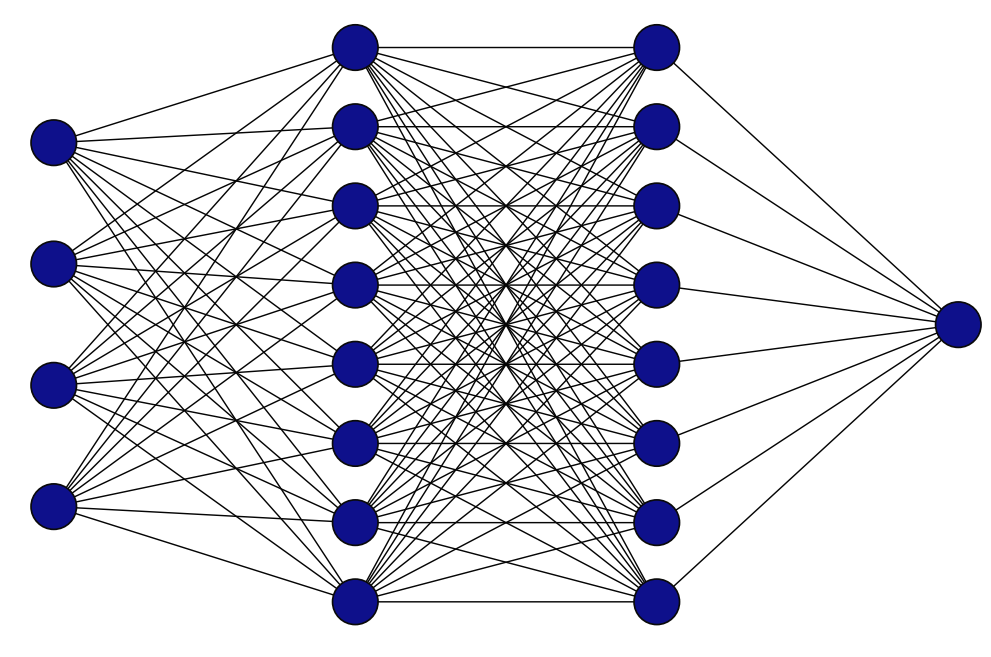

Input Layer $\in \mathbb{R}^{4}$

Hidden Layer $\in \mathbb{R}^{8}$

Hidden Layer $\in \mathbb{R}^{8}$

Output Layer $\in \mathbb{R}^{1}$

Figure 5. Artificial neural network architecture.

\subsubsection{Evolutionary Strategies}

The evolutionary strategies (ES) are stochastic techniques for the numerical optimization of non-linear and non-convex learning problems. This class of ML methodologies is inspired by the biological evolution of individuals due to specific environmental conditions, also referred to as the survival of the fittest. In the context of the PUF technology, this individual is represented by a vector of runtime delays in the circuit components. The algorithm generates random PUF instances that are referred to as parents. They are tested to check the resemblance with the target PUF responses using the fitness function that should be specified by the user. Afterwards, the child instances inherent the parents' characteristics (delay vectors in the case of Arbiter PUFs) with minor random modifications, and the resemblance process is conducted for many generations.

The Covariance Matrix Adaptation Evolutionary Strategy (CMA-ES) [44] is one of the most known ES that performs well on complex optimization problems. This variant uses the covariance matrix to adjust the dependencies between the variables in the normal distribution. Figure 6 illustrates the steps of the CMA-ES technique. The algorithm starts by generating random parent individuals according to the normal distribution. Afterwards, the fittest candidates are selected based on a specific fitness function and the algorithm updates its internal parameters. Finally, a new population is generated based on the previous updates, and the process is repeated until convergence. 


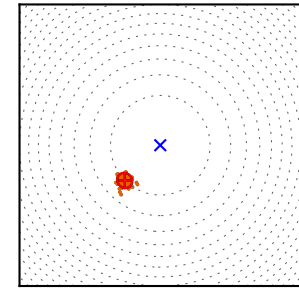

(a) Iteration 1

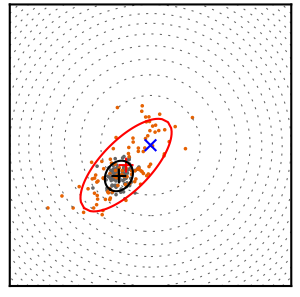

(b) Iteration 3

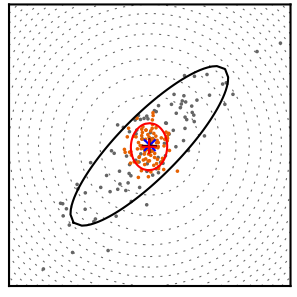

(c) Iteration 5

Figure 6. The optimizationproblem of a two-dimensional linear function using the CMA-ES algorithm. The orange and gray dots represent the distributions of the child and the parent populations.

The CMA-ES technique has been widely used to model complex PUF architectures such as Interpose-PUF [11]. This algorithm was also applied in the work of Becker [45] to attack the two versions of the Slender-PUF $[15,46]$. The adversary targeted the response obfuscation mechanism in order to use the obfuscated CRPs to efficiently model PUF circuit.

\subsubsection{Other Machine Learning Techniques}

Other ML techniques such as decision tree, random forest and naïve Bayes classification have been applied to model the behavior of the PUF circuits [21,47]. These techniques have been adopted to address the signal classification and networking problems [48,49]. However, in the work of Kroger et al. [47], they were demonstrated to be less effective in comparison to the previously described algorithms when using a relatively large dataset of challenge-response pairs (more than $400 \mathrm{CRPs}$ ). On the other hand, these techniques achieved a better accuracy wh training on a small dataset (less than $400 \mathrm{CRPs}$ ).

\section{Model-Based PUF Authentication Procedure}

In this section, we describe the authentication process of an IoT object based on the use of a mathematically clonable PUF based on a number of ML techniques. The procedure consists of multiple entities that participate in verifying the identity of this particular device. These entities constitute two generic architectures that represent the steps of an enrollment protocol. Each of these components are defined and characterized based on the roles and the modules that are specified by the protocol designer. The building block diagrams in these two architectures can help to design and assess independently the system components of these schemes with respect to the adopted threat model. Furthermore, we provide global insights into the enrollment process and the components. This section introduces the insider threat model in the enrollment process, which is usually overlooked by designers. This model aims at assessing the robustness of the protocols against the scenario of leakage of a secret PUF model to an adversary.

\subsection{Enrollment Architectures}

The existing model-based PUF authentication protocols can be classified based on two generic architectures that we refer to them as three-component (3CE) and four-component (4CE) enrollment procedures. As the name states, the former approach requires the existence of three main high-level roles:

- Prover: The IoT object that needs to be enrolled in the network of the user based on the PUF hardware onboard it.

- Communication channel (CC): The communication channel for the components.

- Authentication server (AS): The entity that manages the storage and the accessibility to the PUF model. Furthermore, it performs the enrollment procedure with the prover as the root of trust (RoT) [50] in the authentication process through the chosen communication channel.

This approach typically requires the unauthenticated IoT object to connect to the network of the user to remotely communicate with the authentication server, as illustrated 
in Figure 7. On the other hand, the latter architecture is slightly different, since it exploits a delegated root of trust (RoT) [50] role, referred to as the verifier. The four components of this approach are described as follows:

- Prover: The IoT object that needs to be enrolled in the network of the user based on the PUF hardware onboard it.

- Communication channel (CC): The communication channel for the components.

- Verifier: The designated entity that performs the enrollment procedure with the prover on behalf of the RoT in the authentication process through the chosen communication channel. This role and the the authentication server constitute the chain of trust in the enrollment procedure.

- Authentication server (AS): The entity that manages the storage and the accessibility to the PUF model. Moreover, it adds the enrolled prover to the list of authorized devices to join the network based on the validation of the verifier.

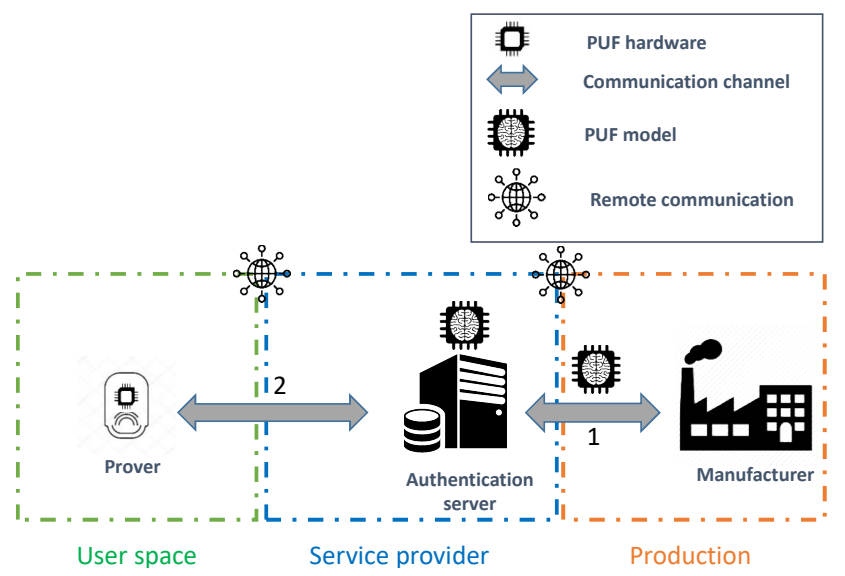

Figure 7. Three-component enrollment procedure.

The delegated root of trust acts as the local challenger of the IoT device, as shown in Figure 8. Therefore, it prevents the risks related to connecting an unauthenticated object to a poorly isolated network. Furthermore, it helps to decrease the communication and computational costs on the server side. Thus, the verifier role enhances the scalability of the enrollment procedure.

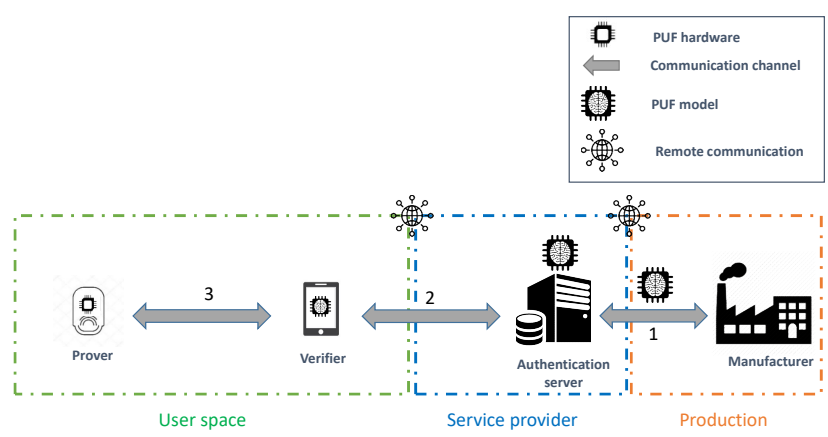

Figure 8. Four-component enrollment procedure.

\subsection{Overview of Components}

In this subsection, we describe the roles and the modules that constitute each component. These generic elementary units serve as building blocks to the previously introduced architectures.

\subsubsection{Prover}

The prover role represents the IoT object that holds the PUF hardware. This secure element represents a means to perform the entity authentication procedure. Depending on 
the adopted enrollment architecture, the IoT device can be given access to the network of the user prior to the authentication process to communicate with the AS, as illustrated in Figure 7. However, in the case of the 4CE approach, the prover is limited to using local communications with the verifier until the successful execution of the enrollment protocol.

The application of a PUF ML model in the protocol design is an admission that this secure element can be mathematically cloned when the adversary has a sufficient number of challenge-response pairs. Therefore, additional protection techniques should be implemented to prevent the attacker from constructing their own precise PUF model. Following the specifications in the work of Maes [51], the added security measures classify this PUF construction as a Controlled PUF. The prover role is established based on three main elementary units, as highlighted in Figure 9, that manage the input-output transformation. The three sub-components are as follows:

- Challenge preparation (CP): The $\mathrm{CP}$ unit is responsible for receiving and preparing the received challenge from the verifier. This part can be split into three main categories:

- Direct reception: The received challenges can be fed directly to the PUF hardware.

- Mutual construction: The prover and the verifier collaborate to compute a common seed to generate the set of challenges. One simple example of this operation is to exchange nonces that are concatenated to find the shared seed value.

- Challenge derivation: The prover receives a single l-bit challenge that is manipulated to extract in total a set of $l$ challenges. As an example of this operation, the receiver can apply a linear-feedback shift register to the received root challenge.

- Challenge verification (CV): The CV unit is responsible for verifying the validity of the challenges that are fed to the PUF hardware. For example, the verification process may aim at ensuring that the received challenges have not been executed before. This technique is considered a means of mitigation against the reliability attack that was proposed in the work of Becker [45].

- Controlled PUF (CPUF): The CPUF unit constitutes the most important component on the prover side. This part is responsible for generating and obfuscating the PUF responses. The CPUF has three main aspects:

i. PUF architecture: The chosen PUF construction to be implemented in the prover.

ii. Reconfigurability: This aspect is only discussed in the case of FPGA. The integrated circuit onboard the prover can be reconfigured by the verifier to impose a specific behavior of the PUF.

iii. Obfuscation technique: The specification of the chosen approach to hide the responses from the adversary to prevent any modeling attacks based on the collected CRPs.

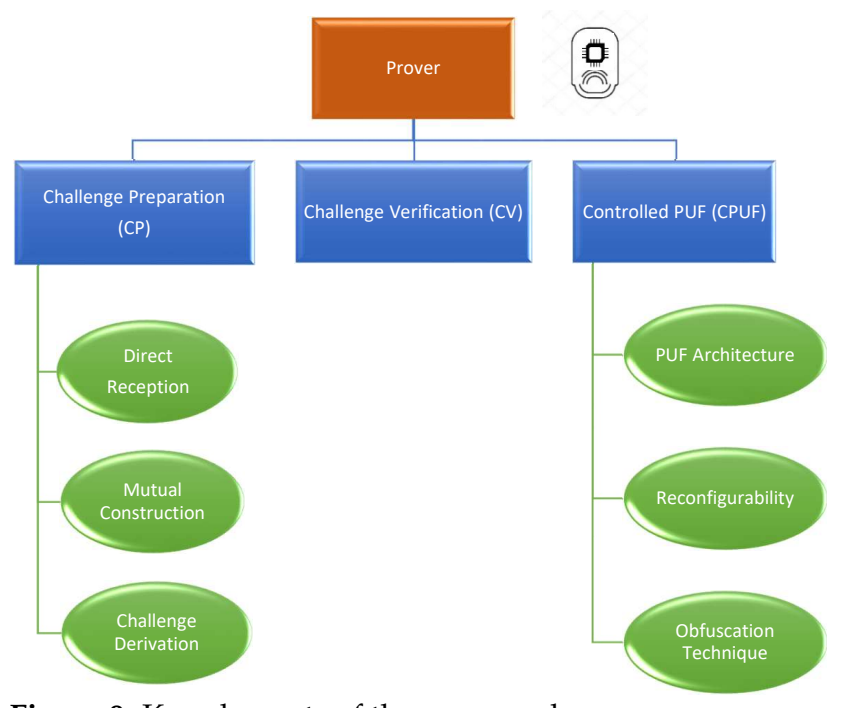

Figure 9. Key elements of the prover role. 


\subsubsection{Verifier}

The verifier role is considered as the local root of trust that initiates the challengeresponse process with the prover, as illustrated in Figure 8. This component plays a crucial role in generating the enrollment challenges and in verifying the validity of the received obfuscated responses. In this context, the verifier takes advantage of the received PUF model from the authentication server to perform the enrollment process, as illustrated in Figure 10. The verification responsibility can be divided into two main parts:

- Response re-computation: The verifier applies the chosen challenges to the PUF model to extract a set of probably approximately correct responses.

- Response verification: This process uses the received responses from the prover and the re-computed values from the PUF model to validate the identity of the sender.

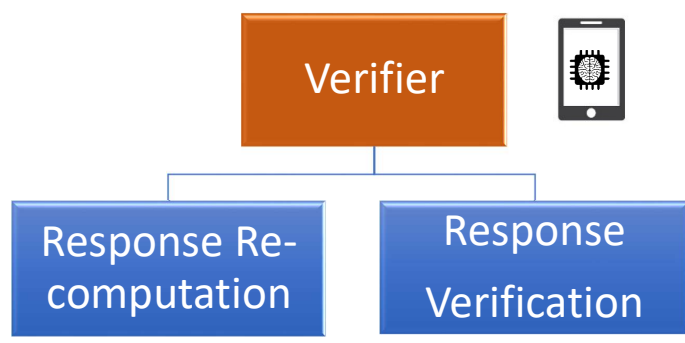

Figure 10. Key elements of the verifier role.

\subsubsection{Authentication Server}

The authentication server is considered as the primary root of trust in both architectures. This component guarantees the integrity, and in most cases, the confidentiality of the PUF model depending on the security properties required of the enrollment protocol. Consequently, the AS can be classified into three categories based on these security guarantees, as shown in Figure 11. The classification of the AS operational mode is described as follows:

- Public database: The authentication server has to guarantee the integrity of the PUF model that can be accessed publicly by any participant.

- Private database: The authentication server has to guarantee the integrity and the confidentiality of the PUF model that can only be accessed by the authorized users.

- Root authenticator: The authentication server stores the PUF model under one of the previous database modes. Furthermore, it fully plays the role of the verifier as introduced in the 3CE architecture.

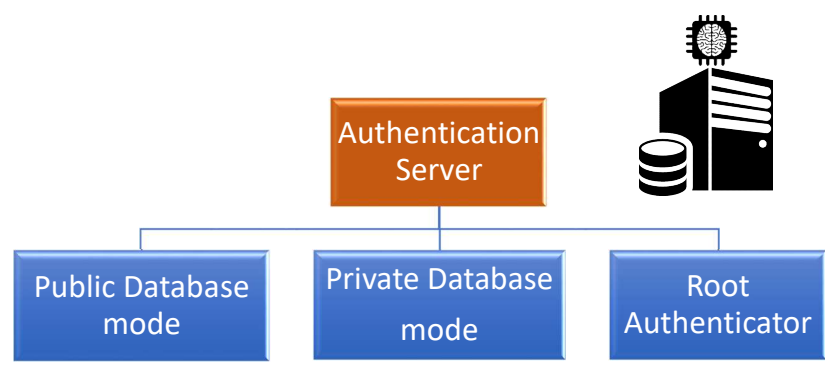

Figure 11. Key elements of the authentication server's role.

\subsubsection{Manufacturer}

The manufacturer plays the initial role of constructing the prover hardware. He extracts enough challenge-response pairs to construct the PUF model and he sends it securely to the authentication server. These actions mark the end of participation of the manufacturer in the enrollment process. 


\subsection{Threat Models}

The adopted threat models in the existing ML model-based PUF authentication protocols can be categorized depending on the accessibility properties of the ML model in question. As described in Section 3.2.3, the private database and root authenticator modes require the authentication server to keep the PUF model a secret and to only provide access to the trusted users. Thus, the adversary cannot get hold of the PUF model, and he can only attack the system through external actions such as eavesdropping or replaying the messages exchanged between the enrollment entities. This attacker falls into the outsider threat category. However, The public database mode assumes that any user can obtain the model without any restrictions. The security of this mode is assured by relying on additional assumptions about the attacker's capabilities. Regarding the adversary's powers over the communication channel, he is able to eavesdrop on the exchanges between the prover and the verifier or the authentication server depending on the adopted enrollment architecture. Furthermore, he can actively query the PUF holder by its own challenges. This action aims at collecting enough CRPs for the attempted model reconstruction attack in the case of the private operational modes. However, the adversary is assumed unable to conduct invasive attacks on the prover software, which guarantees the correctness of the enrollment protocol execution. This assumption can be assured through the use of lightweight integrity verification of IoT systems such as the remote attestation schemes [52,53]. As a consequence, the adopted threat models are classified as follows:

- Public model adversary (Pub-Adv): The goal of the adversary shifts from modeling the PUF hardware to attacking the additional security mechanisms in order to bypass the authentication process. For example, he can focus on reducing the response generation time using the public PUF model to bypass the time-bound assumption.

- Private model adversary (Priv-Adv): The adversary aims at creating a precise PUF model based on the obfuscated challenge-response exchanges. This ML model serves as a tool to predict the correct responses to the challenges of the verifier as a way to enroll malicious devices.

The two previously detailed attacker categories can be further extended to assess the robustness of the enrollment protocol against an adversary that can get hold of the PUF model that is used in the authentication process. This scenario is considered as an insider threat within the information system of a particular organization. The attack is based on an individual with sufficient access privileges who violates the non-disclosure policies by leaking sensitive information, such as the PUF models. These leaks should be impossible to be traced back to this particular individual. This scenario is only applicable in the context of the $4 \mathrm{CE}$ architecture where the verifier might be the source of the leakage, since it represents the role with the least level of trust in comparison with the authentication server. On the other hand, the verifier is assumed to be able to properly perform the authentication process without the risk of fraudulently enrolling malicious devices. This is due to the fact that the enrollment process of a particular device can lead back to the individual responsible once the malicious object is discovered. However, the PUF model, is shared between all the potential operators, which eliminates any possibility of discovering the leakage source.

\section{Enrollment Protocols Analysis}

In this section, we study a selection of model-based PUF enrollment protocols based on the previously identified architectures. The different modules that are applied in the components of these schemes are described and detailed. Afterwards, we provide a security overview of the identified weaknesses in the protocol design and we suggest the adequate mitigation.

\subsection{Time-Bounded Authentication Protocol}

This enrollment scheme was proposed in the work of Majzoobi and Koushanfar [54,55] to target the issue of having a public model architecture of the PUF. The security of the 
protocol is based on the assumption that the time required to generate the responses by PUF hardware is significantly smaller than the time required to predict them using a machine learning model. Thus, it is possible to verify the origin of the received responses by the verifier to avoid any possible ML-based impersonation attacks. The main steps of the time-bound authentication process are illustrated in Figure 12. This proposal is based on the $4 \mathrm{CE}$ architecture and adopts the public adversary threat model, which are described, respectively, in Sections 3.1 and 3.3 .

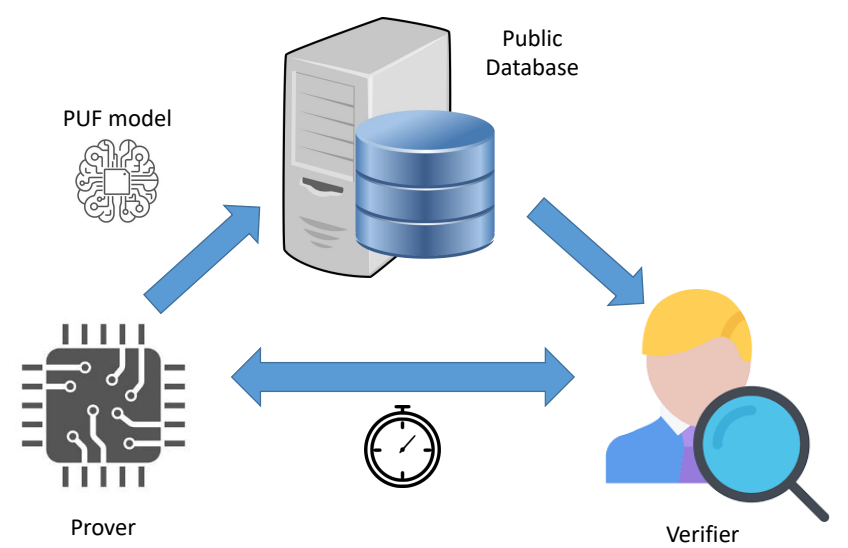

Figure 12. High-Level representation of the time-bound authentication protocol.

\subsubsection{Protocol Components}

\section{Prover}

In this protocol, the operational characteristics of the prover are systematically described based on the following three sub-components:

- Challenge preparation: Direct reception.

- Challenge verification: The received challenges are not verified.

- Controlled PUF:

i. PUF specifications:

- Nature: Electronic.

- $\quad$ Architecture: C-RPUF (Section 2.1.3).

ii. Reconfigurability: This option is fully supported.

iii. Obfuscation technique: This mechanism is not applied. The responses are returned to the verifier without any modification.

\section{Verifier}

The operational characteristics of the verifier are systematically described based on the following two sub-components:

- Response re-computation: The verifier applies the public model that is stored in the authentication server, and the desired reconfiguration to predict the responses of the prover.

- Response verification: The verifier evaluates the execution time of the challengeresponse process. The verification of the PUF output happens only if the responses are received within a pre-fixed time threshold. When the time-bound assumption is satisfied, the response verification process is conducted by a simple bitwise comparison.

\section{Authentication Server}

The AS in the protocol plays the role of a public database. Therefore, the PUF model is also accessible to the adversary. However, the integrity of the stored PUF model is assumed guaranteed. 


\subsubsection{Security Assessment}

Authentication Property. The verifier authenticates the prover only if the time the prover takes to generate the correct response is less than the time-bound threshold.

To handle the public accessibility to the PUF model, the work of Majzoobi and Koushanfar [55] uses a time-bounded method that prevents the prover from applying a PUF model, since it takes more time than just feeding the challenge as an input to the PUF hardware. In addition, the messages containing the configuration bitstream provide insights about the placements of the specific PUF cells to be used in the case of a reconfigurable PUF. However, the adversary is assumed to be unable to reverse-engineer this information, which prevents him from knowing the used PUF configuration. This assumption suggests that the attacker does not have perfect knowledge of the protocol structure, which partially supports the Security Through Obscurity (STO) policy. Thus, this mechanism might be vulnerable to an attack on the distance-bounding protocols $[56,57]$. Therefore, there is a need for a new method to guarantee that the source of the response is indeed the PUF hardware and not the model used. Since the manufacturer constructs the model of the PUF and stores it in a publicly accessed database, the adversary is assumed to be able to obtain it, just as any legitimate user can. In order to prevent the attacker from using the PUF model to respond to the challenge, the verifier applies a time-bound authentication proof to the challenge-response process based on the assumption that the time required for the response simulation is longer than the time required by the hardware PUF. This assumption is only valid if the minimum response simulation time, represented as $t_{m i n}^{\text {sim }}$, is larger than the upper bound delay for generating an authentic response by the hardware that is represented as $\Delta_{\max }$.

The time-bound assumption is based on the computational capabilities of the adversary and the variation in the channel latency to guarantee the correctness of the authentication process. This explains the use of the additional STO assumption about the infeasibility of the attacker decoding the configuration bit-stream, which prevents him from efficiently simulating the behavior of the PUF. In addition, this particular protocol is mainly designed for FPGAs, which makes it unsuitable for the application-specific integrated circuits, such as the majority of the IoT devices. Thus, the reconfigurability technique cannot be applied to thwart the risks of bypassing time-bound authentication.

\subsection{Slender PUF Protocol}

The Slender PUF protocol was proposed in two versions. The conference version was first introduced in the work of Majzoobi et al. [15] to present a new response hiding technique that is based on pattern matching. However, the journal version [46] represents an extension of the response obfuscation through a pseudo-random padding of the selected sub-string. These two proposals are based on the $3 \mathrm{CE}$ architecture, and they adopt the private model adversary. The details of these two terms are described, respectively, in Sections 3.1 and 3.3.

\subsubsection{Protocol Components}

Prover

In this protocol, the operational characteristics of the prover are systematically described based on the following three sub-components:

- Challenge preparation: Mutual construction through a nonce exchange.

- Challenge verification: The received challenges are not verified.

- Controlled PUF:

i. PUF specifications:

- $\quad$ Nature: Electronic.

- $\quad$ Architecture: 4-XOR Arbiter PUF (Section 2.1.2).

ii. Reconfigurability: This option is not supported. 


\section{iii. Obfuscation technique:}

- Conference version [15]: The prover generates a random index ind $\in[0, l-1]$ that represents the first bit of the truncation. Afterwards, he extracts the $l_{\text {sub }}$ bits sequence from the $l$ bit PUF response to the sent challenges. Then, he sends it to the verifier to validate the enrollment procedure.

- Journal version [46]: The prover conducts the same operations to find the substring response as in the conference version. Then, he generates an additional random $\left(l-l_{\text {sub }}\right)$ bit sequence that serves as padding for the substring. Finally, he inserts the truncated response at a random index ind $_{2} \in\left[0, l-l_{\text {sub }}-1\right]$ of the generated circular padding sequence.

\section{Verifier}

The operational characteristics of the verifier are systematically described based on the following two sub-components:

- Response re-computation: The verifier uses the PUF secret model that is stored in the authentication server, to precisely compute the expected hardware response.

- Response verification: The verification phase is the same for the both versions of the protocol. The verifier tries to find a match between the substring and the simulated PUF response through a maximum sequence alignment. The enrollment is validated under two conditions: the substring alignment should produce a match and the hamming distance between the two sequences should be less than a pre-defined threshold. The latter condition is applied to support the noisiness in the PUF responses.

\section{Authentication Server}

The AS in the protocol plays the role of a root authenticator. Therefore, the PUF model is not accessible to the adversary. Consequently, the authentication server has to guarantee the confidentiality and the integrity of the PUF model.

\subsubsection{Security Assessment}

Authentication Property. The authentication is successful if the prover response substring matches at some location in the authentication server's estimated response string within a predefined threshold of time.

The two versions of the Slender PUF protocol were put to the test in the work of Becker [45]. In this experiment, the author applied the CMA-ES [44] machine learning algorithm, detailed in Section 2.2.4. In the case of the attack on the Slender PUF, Becker targeted the main security assumption of the protocol that the adversary can only compromise the protocol by guessing the truncation indexes, $i n d_{1}$ and $i n d_{2}$. This assumption aims to establish that the only possible way to model the PUF hardware is to map the substring response sequence to the corresponding challenges. The proposed attack counters this assumption by using a Pearson correlation coefficient $\operatorname{corr}($.$) [58] as a fitness test between$ the Hamming weights of the generated responses from the parent PUF instances, $H W\left(R_{i}\right)$, and the Hamming weights of the collected substrings, $H W\left(W_{i}\right)$. The choice of this fitness function was motivated by the assumption of the higher the computed correlation, the more accurate the PUF instance. This technique efficiently modeled the protected hardware PUF using different levels of noise and two constructions of PUFs (3-XOR and 4-XOR Arbiter PUF). The added noise was applied to simulate the unreliability percentages of the collected hardware PUF responses. The accuracy of the modeled 4-XOR Arbiter PUF reached $97.2 \%$ using 600,000 noiseless CRPs. However, the additional $29 \%$ noisy responses reduced the accuracy to $92.5 \%$ using $1,200,000$ samples.

\subsection{Noise Bifurcation Protocol}

The noise bifurcation protocol was introduced in the work of Yu et al. [31] to present a novel response hiding technique. The scheme selects only specific responses to be returned 
to the verifier. Thus, the attacker is assumed unable to associate the challenges and their corresponding responses. The proposal is based on the $3 \mathrm{CE}$ architecture, and it adopts the private model adversary. The details of these two terms are described, respectively, in Sections 3.1 and 3.3.

\subsubsection{Protocol Components}

Prover

In this protocol, the operational characteristics of the prover are systematically described based on the following three sub-components:

- Challenge preparation: Mutual construction through a challenge exchange. The master challenges are referred to, respectively, as $C_{p}$ for the one generated by the prover and $C_{v}$ for the one generated by the verifier.

- Challenge verification: The received challenges are not verified.

- Controlled PUF:

i. PUF specifications:

- Nature: Electronic.

- Architecture: 4-XOR Arbiter PUF with multiple derivative challenges (Section 2.1.2).

ii. Reconfigurability: This option is not supported.

iii. Obfuscation technique: The prover generates a random challenge $C_{p}$ that represents the second master challenge. Then, he extracts a set of $m$ challenges from $C_{p}$ and $C_{v}$. The resulting $m$ responses $R \in\{0,1\}^{m}$ is divided into $\frac{m}{d}$ groups of $d$ elements (in [31], $d=2$ ). Afterwards, only one response per group is randomly chosen and they are returned as a reply to the verifier. The previously described obfuscation technique is illustrated in Figure 13.

Prover

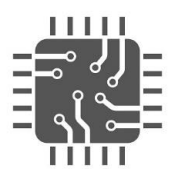

$011100011011 .$.

$\theta 11100 \quad 011011 .$.
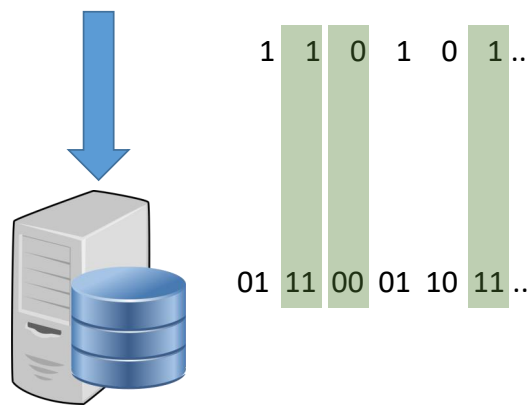

Authentication Server

Figure 13. Noise-Bifurcation obfuscation technique.

\section{Verifier}

The operational characteristics of the verifier are systematically described based on the following two sub-components:

- Response re-computation: The verifier uses the PUF secret model to precisely compute the expected hardware response.

- Response verification: The verifier reconstructs the $\frac{m}{d}$ groups using the recomputed responses. Then, he selects the matching responses with the same group and performs 
the comparison with received results, as highlighted in green in Figure 13. The authentication is successful only when the hamming distance between the selected and the received responses is below a pre-defined tolerance threshold.

\section{Authentication Server}

The AS in the protocol plays the role of a root authenticator. Therefore, the PUF model is not accessible to the adversary. Consequently, the authentication server has to guarantee the confidentiality and the integrity of the PUF model. Most importantly, the delivery of the secret model should be only allowed for the authorized users. Additionally, the AS plays the role of the verifier in the enrollment process.

\subsubsection{Security Assessment}

Authentication Property. The prover is authentic if the number of mismatched bits, that are computed by the authentication server, are lower than a pre-defined threshold.

The noise bifurcation protocol have been assessed in the work of Tobisch and Becker [59] through the re-execution of the evaluation methodologies presented in the original paper [31]. The modeling attack focuses on the full-response replication strategy to construct the CRP dataset. This technique aims at associating each bit response with the $d$ challenges of the corresponding group. However, this assessment revealed some contradictions with the original results published in [31] that is due to the lack of specifications about the applied PUF construction. The original work exploited a XOR Arbiter PUF where each XOR stage receives a random unique challenge. This specific architecture is considered as an additional countermeasure that has not been clearly described. The evaluation of the noise bifurcation technique on a classical PUF construction, where the same challenge is applied to all the stages, reveled that the obfuscation scheme does not prevents the adversary from modeling the PUF. The attack was conducted using the logistic regression model with a considerable number of CRPs that depends on the number of XOR stages with an accuracy that varies between $84 \%$ and $92 \%$. The details of the applied ML technique are described in Section 2.2.1.

\subsection{OB-PUF Protocol}

The OB-PUF protocol was introduced in the work of Gao et al. [60] to present a challenge obfuscation technique. The main objective behind the scheme is to prevent the adversary from constructing a sound CRP dataset that is, eventually, used to model the PUF behavior. On the other hand, the legitimate verifier holds the PUF model that is used to authenticate the prover based on the received responses. The proposal is based on the $3 \mathrm{CE}$ architecture and it adopts the private model adversary. The details of these two terms are described, respectively, in Sections 3.1 and 3.3.

\subsubsection{Protocol Components}

\section{Prover}

In this protocol, the operational characteristics of the prover are systematically described based on the following three sub-components:

- Challenge preparation: Direct reception.

- Challenge verification: The received challenges are not verified.

- Controlled PUF:

i. PUF specifications:

- Nature: Electronic.

- $\quad$ Architecture: Arbiter PUF (Section 2.1.1).

ii. Reconfigurability: This option is not supported.

iii. Obfuscation technique: The prover receives the obfuscated challenge $C_{O B} \in$ $\{0,1\}^{l-k}$ that is sent by the verifier where $l$ is the challenge bit-length (e.g., 
$l=64)$ and $k$ is the number of the obfuscated bits. Afterwards, he randomly chooses the pattern of the additional $k$ bits and executes them using the PUF hardware to obtain a $n$-bit response $R$ where $n$ is the number of Arbiter PUF instances onboard the prover. The pattern is a set of $k$ pre-defined bit values and indices that are used as a padding to the obfuscated challenge, as highlighted in Figure 14. The response $R$ is, then, returned to the verifier.

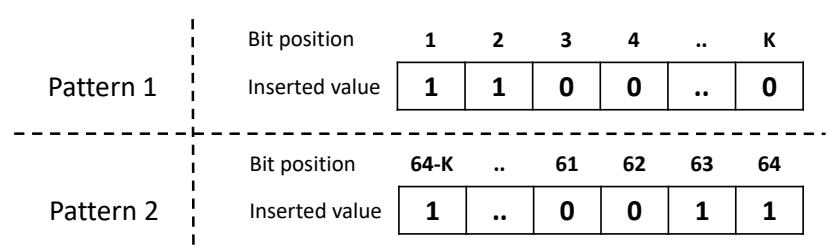

Figure 14. Two pattern examples that might be added to the obfuscated challenge.

\section{Verifier}

The operational characteristics of the verifier are systematically described based on the following two sub-components:

- Response re-computation: The verifier uses the PUF secret model to compute all the possible responses of the obfuscated challenge based on all the pre-defined padding patterns.

- Response verification: The verifier compares the received response with all the predicted responses to authenticate the prover.

\section{Authentication Server}

The AS in the protocol plays the role of a root authenticator. Therefore, the PUF model is not accessible to the adversary. Consequently, the authentication server has to guarantee the confidentiality and the integrity of the PUF model. Most importantly, the delivery of the secret model should be only allowed for the authorized users. Additionally, the AS plays the role of the verifier in the enrollment process.

\subsubsection{Security Assessment}

Authentication Property. The authenticity of the prover is established if the candidate emulated response for the given obfuscated challenge $C_{O B}$ is the same as the received response $R$.

The security of the OB-PUF protocol was compromised in the work of Delvaux [61]. The attack strategy is based on the direct interaction with the prover that is holding the PUF. The main objective of the adversary is to search for the obfuscated challenges $C_{O B}$ that generate similar results. This process is conducted through the repetitive execution of the same obfuscated challenges for a specific number of times and the assessment of the resulting responses. The collected CRPs serve as a dataset to construct the ML model of the PUF using logistic regression. The details of the applied ML technique are described in Section 2.2.1.

The original work [60] claimed that the adversary cannot exceed the accuracy limit of $72 \%$ even after collecting $10^{6}$ random CRPs which is not sufficient to bypass the authentication. However, the described strategy provided the attacker with the ability to reach an $85 \%$ accuracy using the same ML technique. Afterwards, the attacker extended their strategy to use the constructed model to build a new dataset using uniformly distributed challenges. This procedure increased the accuracy of the adversarial model to reach $95 \%$. This attack could have been mitigated through the application of a challenge verification procedure on the prover side that eliminates the repetitive execution of the same obfuscated challenge. This could be done by the use of an approximate set membership test such as the XOR filter [62]. 


\subsection{Lightweight PUF-Based Authentication Protocol}

The lightweight PUF authentication protocol was introduced in the work of Yilmaz et al. [63] to present a suitable enrollment protocol for the resource-constrained devices. The main objective behind the scheme is to reduce the power and memory consumption with respect to the legacy IoT protocol DTLS handshake authentication. The proposal is based on the 4CE architecture and it adopts the private model adversary. The details of these two terms are described, respectively, in Section 3.1 and in Section 3.3.

\subsubsection{Protocol Components}

\section{Prover}

In this protocol, the operational characteristics of the prover are systematically described based on the following three sub-components:

- Challenge preparation: Direct reception.

- Challenge verification: The received challenges are not verified.

- Controlled PUF:

i. PUF specifications:

- $\quad$ Nature: Electronic.

- $\quad$ Architecture: Arbiter PUF (Section 2.1.1).

ii. Reconfigurability: This option is not supported.

iii. Obfuscation technique: The prover uses the RC5 encryption scheme [64] to encrypt the MAC address of the device with the response of the PUF $R$. The returned value of the prover is formulated as $R C 5(M A C, R \oplus T)$ where the $T$ parameter is the timestamp which guarantee the freshness of the obfuscation procedure.

\section{Verifier}

- Response re-computation: The verifier uses the PUF secret model to precisely compute the expected hardware response.

- Response verification: The verifier predicts the PUF response through the use of the secret model. Then, he computes the expected output value using the predicted PUF response and the timestamp. Afterwards, he compares the two ciphertexts to validate the authentication process.

\section{Authentication Server}

The AS in the protocol plays the role of a private database. Therefore, the PUF model is not accessible to the adversary. Consequently, the authentication server has to guarantee the confidentiality and the integrity of the PUF model. Most importantly, the delivery of the secret model should be only allowed for the authorized users.

\subsubsection{Security Assessment}

Authentication Property. The prover is authenticated if the verifier validates the received RC5 ciphertext using the PUF model response and the timestamp.

The obfuscation technique is based on the RC5 encryption scheme. The security of the procedure is based on the infeasibility to access the PUF responses by an adversary that does not have the accurate model. However, this encryption scheme has requirements regarding the length of the applied key (suggested 128 bits) which is not clearly the case in the original protocol simulation. One study [63] implemented the authentication scheme using a PUF architecture that provide response bit-lengths that vary, respectively, between 16 and 32 bits. Thus, the confidentiality of the sent ciphertext might be compromised through the correlation attack [65] or the timing attack [66]. In addition, the use of an encryption scheme to obfuscate the PUF response without error-correcting codes affects drastically the usability of the protocol. This is due to the non-ideal reliability of the PUF hardware that might produce bit-flips in the responses. Consequently, these incidents 
result in errors in the decryption process on the verifier side. Furthermore, the PUF model predictions might not be always $100 \%$ accurate which ruins the de-obfuscation process.

\subsection{RF-PUF Protocol}

The RF-PUF protocol was introduced in the work of Chatterjee et al. [26] to present an ANN-based process to authenticate the wireless nodes. The details of the applied ML technique are described in Section 2.2.3.

Similar to the concept of the hardware PUFs, the proposal uses the effects of inherent variation on radio-frequency properties of the wireless transmitters Tx (provers). The detection is based on a machine learning model at the receiver side $\mathrm{Rx}$ (verifier). The main objective behind the scheme is to distinguish between the signals received by the provers in order to uniquely identify them, as illustrated in Figure 15. The proposal is based on the $4 \mathrm{CE}$ architecture and it adopts the private model adversary. The details of these two terms are described, respectively, in Sections 3.1 and 3.3.

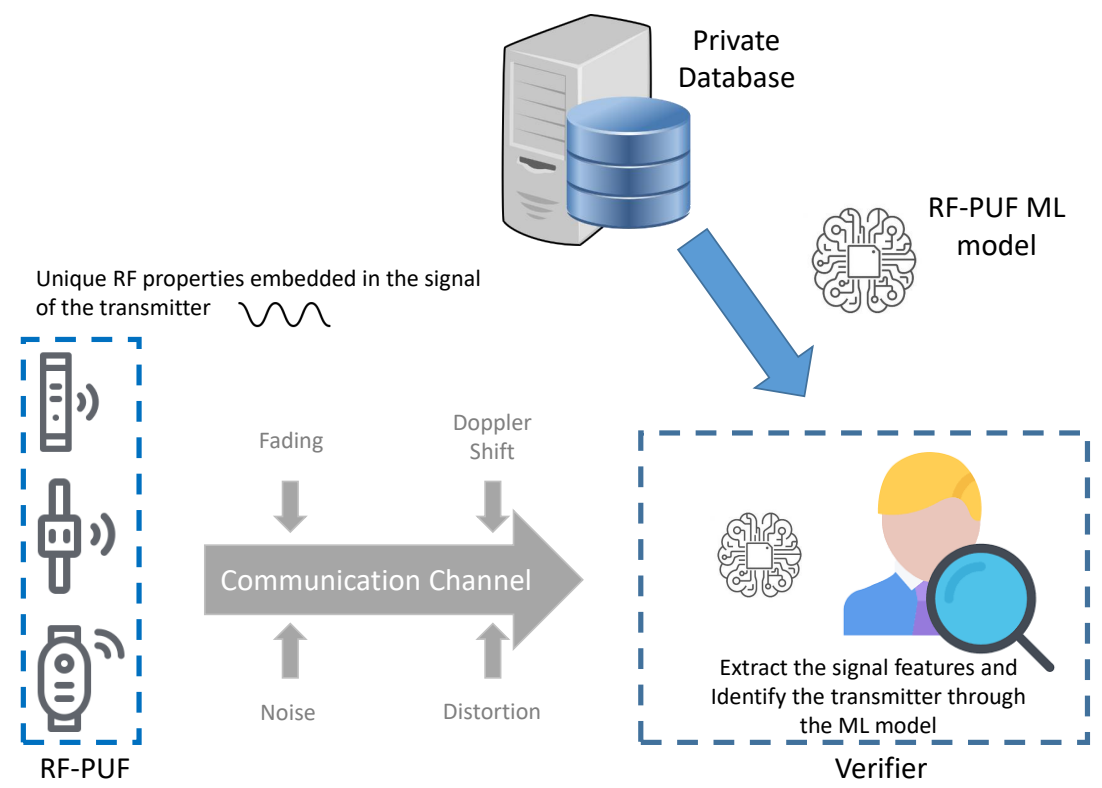

Figure 15. High-Level representation of the RF-PUF protocol.

\subsubsection{Protocol Components}

\section{Prover}

In this protocol, the operational characteristics of the prover are systematically described based on the following three sub-components:

- Challenge preparation: Direct reception.

- Challenge verification: The received challenges are not verified.

- Controlled PUF:

i. PUF specifications:

- $\quad$ Nature: Non-electronic.

- $\quad$ Architecture: RF-PUF.

ii. Reconfigurability: This option is not supported.

iii. Obfuscation technique: This mechanism is not applied. The responses are returned to the verifier without any modification.

\section{Verifier}

- Response re-computation: This option is not supported.

- Response verification: The verifier identifies the transmitters through their radio signatures and the ANN model. 


\section{Authentication Server}

The AS in the protocol plays the role of a private database. Therefore, the PUF model is not accessible to the adversary. Consequently, the authentication server has to guarantee the confidentiality and the integrity of the PUF model. Most importantly, the delivery of the secret model should be only allowed for the authorized users.

\subsubsection{Security Assessment}

Authentication Property. The prover is authenticated if the verifier validates the RF signature of the prover through the ANN model.

The RF-PUF is based on a machine learning model that identifies specific communication nodes through a set of propagation properties (local oscillator frequency, channel information, DC offset and I-Q mismatch on the transmitter side). The model is trained using a dataset of challenge-response pairs that are collected from a group of different transmitters. The challenge is a pre-defined bit-sequence that is transmitted to the receiver node. The corresponding response is represented as a set of propagation features that are extracted from the challenge transmission. The model is trained to distinguish between a number of transmitters with a high accuracy under varying channel conditions. The RF-PUF protocol can authenticate up to 10,000 devices with an accuracy of 99\%. However, the decommissioning of the deployed devices poses a serious threat to the security of the protocol. This is explained by the unfeasability of removing a specific device from the list of accepted identities. This operation can be conducted by retraining the model from scratch without using the CRP dataset of that decommissioned device which is computationally costly, especially when managing a big number of IoT objects.

The authors in [26] have discussed the possibility of facing an attacker that tries to mimick a specific transmitter through the use of a machine learning model. The adversarial model in question intends to produce the same transmission signature as the target transmitter through the collection of a sufficient number of CRPs. The paper argues that the adversary cannot associate the collected CRPs to their corresponding identities when he eavesdrops on a multi-device environment. Therefore, the attacker requires a larger dataset to enhance the accuracy of their unsupervised learning model. However, the unidentified CRPs can be indexed when we take under consideration the insider threat scenario where an adversary can obtain the ANN identification model. Thus, it transforms back the problem into a supervised learning procedure that facilitates the mimicking attack.

\subsection{Set-Based Obfuscation Protocol}

The Set-Based Obfuscation protocol was introduced in the work of Zhang and Shen [67] to present an obfuscation technique that resists the existing ML modeling attacks. The introduced methodology relies on the use of a secret set of CRPs that is stored on the authentication server and on the prover. These obfuscation CRPs serve as a way to modifiy the inputs and outputs of the PUF to reinforce the complexity of the PUF mapping function. The proposal is based on the 3CE architecture and it adopts the private model adversary. The details of these two terms are described, respectively, in Sections 3.1 and 3.3.

\subsubsection{Protocol Components}

Prover

In this protocol, the operational characteristics of the prover are systematically described based on the following three sub-components:

- Challenge preparation: Direct reception.

- Challenge verification: The received challenges are not verified.

- Controlled PUF:

i. PUF specifications:

- Nature: Electronic.

- $\quad$ Architecture: Arbiter PUF Section 2.1.1. 
ii. Reconfigurability: This option is not supported.

iii. Obfuscation technique: Random set-based obfuscation (RSO). The obfuscation challenges are stored in the Non-Volatile Memory (NVM). The prover selects randomly two challenges from a set $K$ to be applied to the PUF in order to generate the obfuscation keys, $\mathrm{Key}_{i}$ and $\mathrm{Key}_{j}$. Afterwards, the received challenges are XORed with $K e y_{i}$ to modify the input $C^{\prime}$. Furthermore, the output $R^{\prime}$ is XORed with $K e y_{j}$. The computed response $\widehat{R}$ is split into two $\frac{n}{2}$-bit responses $\left(\widehat{R_{a}}, \widehat{R_{b}}\right)$ where $n$ is the bitlength of $\widehat{R}$. Finally, the $\widehat{R_{b}}$ response is transmitted to the verifier.

\section{Verifier}

- Response re-computation: The verifier uses the PUF secret model and the set of obfuscation CRPs to compute all the potential responses.

- Response verification: The verifier compares the received response to the computed set of potential responses. The enrollment is successful if the verifier finds two responses where the number of mismatched bits is less than a pre-defined threshold.

\section{Authentication Server}

The AS in the protocol plays the role of a root authenticator. Therefore, the PUF model is not accessible to the adversary. Consequently, the authentication server has to guarantee the confidentiality and the integrity of the PUF model. Most importantly, the delivery of the secret model should be only allowed for the authorized users. Additionally, the AS plays the role of the verifier in the enrollment process.

\subsubsection{Security Assessment}

Authentication Property. The prover is authenticated if the authentication server finds a candidate simulated response that has a bit-rate mismatch with the received prover response which is lower than a pre-defined threshold.

The RSO obfuscation technique has been proven resilient against the existing ML modeling attacks, such as LR, SVM, ANN and CMA-ES. The modeling accuracy has been reduced to a limit closer to $50 \%$, which is equivalent to a random guess. This technique requires the storage of the obfuscation CRPs on both the AS and the prover. Each obfuscation challenge consists of a list of $n$ sub-challenges. Therefore, the total number of used sub-challenges is $m \times n$. Thus, the storage space is estimated to be $m \times n \times n$ bits. In order to achieve the maximum level of security that the protocol can offer, the recommended number of bits according to the original paper [67] is $n=128$. Thus, the required storage space is directly dependent on the number of the obfuscation challenges $m$ that is controlled by the user. For example, in the case $m=1000$, the required NVM memory space is 16 Megabits, which is not suitable for resource-constrained devices. On the other hand, the use of less obfuscation challenges may affect the performance of the RSO scheme against the modeling attacks. This is explained by the application of the setupdating mechanism [67], which updates the set of obfuscation challenges located in set $K$. Therefore, there is a need to study the effect of the repetitive use of obfuscation challenges.

\section{Discussion and Future Research Directions}

In this section, we discuss the highlighted results in Table 1. The state-of-the-art modelbased PUF protocols have adopted one of the two architectures presented in Section 3.1. The $3 \mathrm{CE}$ architecture, which is used by a number of protocols in Section 4, requires the IoT object (prover) to communicate directly with the remote authentication server. Thus, the obligation to connect the device to the network prior to the authentication procedure presents a potential threat. In addition, this centralized architecture relies on the AS as the root authenticator. Therefore, it increases the workload for the server and limits the scalability in comparison with the decentralized version that is the $4 \mathrm{CE}$ architecture. 
However, the delivery of the PUF model to the verifier nodes to perform the authentication can result in the leakage of this secret.

This insider threat is justified by the risk of delegating the sensitive enrollment information to a trusted device with a lower level of security compared to the AS. The existing 4CE enrollment protocols described in Section 4 have not taken in consideration this insider threat model. However, the time-bound authentication protocol, as described in Section 4.1, has demonstrated constrained resistance based on the reconfigurability parameter, the attacker computational power and the characteristics of the used communication channel. In addition, we have studied the discovered vulnerabilities in the existing model-based PUF enrollment protocols that affect the ousider threat resistance. As detailed in Section 4, a number of these attacks are the results of weakness in constructing the response obfuscation technique or the use of a vulnerable cryptographic scheme. However, some other vulnerabilities are the consequences of the lack of a challenge verification mechanism that would verify the validity of the received challenges by the prover, as in the case of the OB-PUF protocol.

The design process of the model-based PUF enrollment protocol can be enhanced through the use of our proposed architectures and the attacker models. The building block diagrams in the $3 \mathrm{CE}$ and $4 \mathrm{CE}$ structure can help future researchers to design and assess the components of the protocols independently. Furthermore, they facilitate creating a mitigation procedure related to an attack on a specific component of the authentication process. We have shown an example of an attack on the obfuscation technique of the OBPUF protocol that could have been mitigated through the implementation of a challenge verification component. Unfortunately, in our study we noticed that this component is generally overlooked by protocol designers, as shown in Table 1. Moreover, the insider threat resistance is still an open research question, since it cannot be fully guaranteed by the existing model-based PUF enrollment protocols, as illustrated in Table 1.

In the insider threat scenario, the leaked PUF ML model can be used to successfully bypass the authentication procedure. Therefore, there is a need for an identification mechanism to recognize the use of that specific model during an enrollment session. The use of ML watermarking techniques [69,70] represents a promising solution to performing this particular task. However, all of these existing watermarking methods target mainly the digital media classification models (images, videos or sounds), and they cannot be used for PUF models. This is explained by the nature of the PUF circuit, which takes as an input a random bit sequence challenge. For instance, the application of an out-of-distribution input challenge as a trigger (the trigger is an input sample that is intentionally assigned a wrong label by the watermarked model) cannot be adopted in our case because every combination of bits belongs to the challenge set $\{0,1\}^{l}$. Moreover, any kind of modification to the challenge bit sequence directly modifies the labeled response, and consequently affects the prediction accuracy of the PUF model. This is explained by the difficulty of changing the high likelihood response prediction of a random challenge without reducing the overall performance of the watermarked model. Thus, it is no longer possible to learn the correct behavior of the PUF circuit. Consequently, there a need for a specifically crafted watermarking technique for the case of the binary output PUF models. 
Table 1. Summary of the studied enrollment protocols.

\begin{tabular}{|c|c|c|c|c|c|c|c|c|c|c|c|}
\hline \multirow{3}{*}{ Protocol } & \multirow{3}{*}{ Architecture } & \multicolumn{5}{|c|}{ Prover } & \multicolumn{2}{|c|}{ Verifier } & \multirow{3}{*}{$\begin{array}{l}\text { Authentication } \\
\text { Server }\end{array}$} & \multicolumn{2}{|c|}{ Security Assessment } \\
\hline & & \multirow{2}{*}{$\begin{array}{l}\text { Challenge } \\
\text { Preparation }\end{array}$} & \multirow{2}{*}{$\begin{array}{l}\text { Challenge } \\
\text { Verification }\end{array}$} & \multicolumn{3}{|c|}{ CPUF } & \multirow{2}{*}{$\begin{array}{c}\text { Response } \\
\text { Re-Computation }\end{array}$} & \multirow{2}{*}{$\begin{array}{c}\text { Response } \\
\text { Verification }\end{array}$} & & \multirow{2}{*}{$\begin{array}{c}\text { Outsider Threat } \\
\text { Resistance }\end{array}$} & \multirow{2}{*}{$\begin{array}{c}\text { Insider Threat } \\
\text { Resistance }\end{array}$} \\
\hline & & & & $\begin{array}{c}\text { PUF } \\
\text { Construction } \\
\end{array}$ & Reconfigurability & $\begin{array}{c}\text { Obfuscation } \\
\text { Technique }\end{array}$ & & & & & \\
\hline Time-bounded Authentication Protocol $[54,68]$ & $4 \mathrm{CE}$ & Direct Reception & $\mathrm{n} / \mathrm{a}$ & C-RPUF & Yes & $\mathrm{n} / \mathrm{a}$ & Yes & $\begin{array}{l}\text { Time-bound Verification } \\
\text { Bitwise Comparison }\end{array}$ & Public Database & Partially Yes & Partially Yes \\
\hline Slender PUF Protocol $[15,46]$ & $3 \mathrm{CE}$ & Mutual Construction & $\mathrm{n} / \mathrm{a}$ & 4-XOR Arbiter PUF & No & Substring Matching & Yes & Response Correlation & Root Authenticator & No & - \\
\hline Noise Bifurcation Protocol [31] & $3 \mathrm{CE}$ & Mutual Construction & $\mathrm{n} / \mathrm{a}$ & 4-XOR Arbiter PUF & No & Noise Bifurcation & Yes & Bitwise Comparison & Root Authenticator & No & - \\
\hline OB-PUF Protocol [60] & $3 \mathrm{CE}$ & Direct Reception & $\mathrm{n} / \mathrm{a}$ & Arbiter PUF & No & Obfuscated Challenge Insertion & Yes & Bitwise Comparison & Root Authenticator & No & - \\
\hline $\begin{array}{c}\text { Lightweight PUF-Based } \\
\text { Authentication Protocol [63] }\end{array}$ & $4 \mathrm{CE}$ & Direct Reception & $\mathrm{n} / \mathrm{a}$ & Arbiter PUF & No & Encryption & Yes & Ciphertext Comparison & Private Database & Partially Yes & No \\
\hline Set-Based Obfuscation Protocol [67] & $3 \mathrm{CE}$ & Direct Reception & $\mathrm{n} / \mathrm{a}$ & Arbiter PUF & No & Random Set-based Obfuscation & Yes & Bitwise Comparison & Root Authenticator & Yes & - \\
\hline
\end{tabular}




\section{Conclusions}

In this survey, we have focused on the usage of ML models of PUF circuits for performing the enrollment process due to their scalability advantages. The use of a mathematically clonable PUF requires the adoption of additional security measures to prevent the modeling attacks through the collection of CRPs. This operation is considered quite complex to address without having a clear idea of the different entities of the protocol and their respective components. Therefore, we have introduced two enrollment architectures that map the different nodes participating in the authentication process of the IoT devices. We have studied a selection of model-based PUF enrollment protocols and we have outlined their security limitations with respect to the identified design flaws.

The proposed architectures facilitate the mitigation of some of the highlighted weakness by modifying vulnerable components in the protocol design. The building block diagrams in the 3CE and 4CE structures can help future researchers to design and independently assess the system components of the protocols. The resiliency of the selected enrollment schemes has been assessed against an insider threat within the organization.

This study yielded that these protocols cannot fully guarantee the security of the enrollment procedure when the PUF model is leaked to the adversary. Thus, there is a need for an identification mechanism to recognize the use of that specific ML model during an enrollment session instead of the legitimate hardware. The use of a distancebounding protocol with a fixed time-bound has been previously proposed to distinguish between the use of a PUF hardware and an ML model. However, this technique requires the accurate estimation of a dynamic time-bound that is based on the characteristic of the communication channel and the complexity of the used PUF circuit. In addition, the use of a watermarking technique in the PUF model can be a potential solution to the information leakage problem. This attack scenario is generally overlooked by protocol designers, and mitigation of insider threats is still an open discussion.

Author Contributions: Conceptualization, S.K. and J.L.; Methodology, S.K.; Validation, A.V., I.G. and J.M.; Investigation, S.K.; Resources, S.K.; Original Draft Preparation, S.K.; Review, J.L. and A.V. and I.G. and J.M. and P.U.; Editing, S.K. and J.L.; Visualization, S.K.; Supervision, J.L. and A.V. and I.G. and J.M.; Project Administration, J.L. and P.U.; Funding Acquisition, J.L. and P.U. All authors have read and agreed to the published version of the manuscript.

Funding: This research was funded by the SEIDO lab (The joint research laboratory for Security and Internet of Things between EDF R\&D and Télécom Paris) and the National Association for Research and Technology under grant number 2018/1810.

Institutional Review Board Statement: Not applicable.

Informed Consent Statement: Not applicable.

Data Availability Statement: Not applicable.

Conflicts of Interest: The authors declare no conflict of interest.

\section{Abbreviations}

The following abbreviations are used in this manuscript:

$\begin{array}{ll}\text { ANN } & \text { Artificial Neural Network } \\ \text { AS } & \text { Authentication Server } \\ \text { CC } & \text { Communication Channel } \\ \text { CMA-ES } & \text { Covariance Matrix Adaptation-Evolutionary Strategies } \\ \text { CP } & \text { Challenge Preparation } \\ \text { CPUF } & \text { Controlled PUF } \\ \text { CRP } & \text { Challenge-Response Pair } \\ \text { CV } & \text { Challenge Verification } \\ \text { DAPUF } & \text { Double Arbiter PUF }\end{array}$




$\begin{array}{ll}\text { ES } & \text { Evolutionary Strategies } \\ \text { ETSI } & \text { European Telecommunications Standards Institute } \\ \text { FPGA } & \text { Field-Programmable Gate Array } \\ \text { IoT } & \text { Internet of Things } \\ \text { LR } & \text { Logistic Regression } \\ \text { LR-PUF } & \text { Logically Reconfigurable PUF } \\ \text { ML } & \text { Machine Learning } \\ \text { MLP } & \text { Multi-Layer Perceptron } \\ \text { NIST } & \text { National Institute of Standards and Technology } \\ \text { NVM } & \text { Non-Volatile Memory } \\ \text { OTP } & \text { One-Time Password } \\ \text { Priv-Adv } & \text { Private Model Adversary } \\ \text { PUF } & \text { Physical Unclonable Function } \\ \text { Pub-Adv } & \text { Public Model Adversary } \\ \text { RoT } & \text { Root of Trust } \\ \text { RSO } & \text { Random Set-based Obfuscation } \\ \text { SLP } & \text { Single Layer Perceptron } \\ \text { STO } & \text { Security Through Obscurity } \\ \text { SVM } & \text { Support Vector Machine } \\ \text { 3CE } & \text { Three-Component Enrollment } \\ \text { 4CE } & \text { Four-Component Enrollment } \\ & \end{array}$

\section{References}

1. Caro, F.; Sadr, R. The Internet of Things (IoT) in retail: Bridging supply and demand. Bus. Horizons 2019, 62, 47-54. [CrossRef]

2. Fagan, M.; Fagan, M.; Megas, K.N.; Scarfone, K.; Smith, M. IoT Device Cybersecurity Capability Core Baseline; US Department of Commerce, National Institute of Standards and Technology: Gaithersburg, MD, USA, 2020.

3. Cyber Security for Consumer Internet of Things: Baseline Requirements; Standard ETSI EN 303645 v2.1.1, European Telecommunications Standards Institute: Sophia Antipolis, France, 2020.

4. Guin, U.; Singh, A.; Alam, M.; Cañedo, J.; Skjellum, A. A Secure Low-Cost Edge Device Authentication Scheme for the Internet of Things; IEEE: Pune, India, 2018; pp. 85-90. [CrossRef]

5. Hummen, R.; Ziegeldorf, J.H.; Shafagh, H.; Raza, S.; Wehrle, K. Towards Viable Certificate-Based Authentication for the Internet of Things. In Proceedings of the 2nd ACM Workshop on Hot Topics on Wireless Network Security and Privacy, Budapest, Hungary 19 April 2013; Association for Computing Machinery: New York, NY, USA, 2013; pp. 37-42. [CrossRef]

6. Mathur, S.; Miller, R.; Varshavsky, A.; Trappe, W.; Mandayam, N. Proximate: Proximity-based secure pairing using ambient wireless signals. In Proceedings of the 9th International Conference on Mobile Systems, Applications, and Services, Bethesda, MD, USA, 28 June-1 July 2011; pp. 211-224.

7. Khalfaoui, S.; Leneutre, J.; Villard, A.; Ma, J.; Urien, P. COOB: Hybrid Secure Device Pairing Scheme in a Hostile Environment. In International Conference on Security and Privacy in Communication Systems; Springer: Berlin/Heidelberg, Germany, 2020; pp. 419-438.

8. Jennings, C. Transitive Trust Enrollment for Constrained Devices. 2012. Available online: http://www.lix.polytechnique.fr/ hipercom/SmartObjectSecurity/papers/CullenJennings.pdf (accessed on 12 December 2020).

9. Asim, M.; Guajardo, J.; Kumar, S.S.; Tuyls, P. Physical unclonable functions and their applications to vehicle system security. In Proceedings of the VTC Spring 2009-IEEE 69th Vehicular Technology Conference, Barcelona, Spain, 26-29 April 2009; pp. 1-5.

10. Weimerskirch, A.; Paar, C.; Wolf, M. Cryptographic component identification: Enabler for secure vehicles. In Proceedings of the IEEE Vehicular Technology Conference, Dallas, TX, USA, 19-22 September 1999; Volume 62, p. 1227.

11. Nguyen, P.H.; Sahoo, D.P.; Jin, C.; Mahmood, K.; Rührmair, U.; van Dijk, M. The Interpose PUF: Secure PUF Design against State-of-the-art Machine Learning Attacks. IACR Trans. Cryptogr. Hardw. Embed. Syst. 2019, 2019, 243-290. [CrossRef]

12. Machida, T.; Yamamoto, D.; Iwamoto, M.; Sakiyama, K. A New Arbiter PUF for Enhancing Unpredictability on FPGA. Sci. World J. 2015, 2015, 864812. [CrossRef]

13. Rührmair, U.; Sehnke, F.; Sölter, J.; Dror, G.; Devadas, S.; Schmidhuber, J. Modeling attacks on physical unclonable functions. In Proceedings of the 17th ACM Conference on Computer and Communications Security, Chicago, IL, USA, 4-8 October 2010; pp. 237-249.

14. Wisiol, N.; Mühl, C.; Pirnay, N.; Nguyen, P.H.; Margraf, M.; Seifert, J.P.; van Dijk, M.; Rührmair, U. Splitting the Interpose PUF: A Novel Modeling Attack Strategy. IACR Trans. Cryptogr. Hardw. Embed. Syst. 2020, 2020, 97-120. [CrossRef]

15. Majzoobi, M.; Rostami, M.; Koushanfar, F.; Wallach, D.S.; Devadas, S. Slender PUF protocol: A lightweight, robust, and secure authentication by substring matching. In Proceedings of the 2012 IEEE Symposium on Security and Privacy Workshops, San Francisco, CA, USA, 24-25 May 2012; pp. 33-44. 
16. Liang, W.; Xie, S.; Long, J.; Li, K.C.; Zhang, D.; Li, K. A double PUF-based RFID identity authentication protocol in service-centric internet of things environments. Inf. Sci. 2019, 503, 129-147. [CrossRef]

17. Pour, A.A.; Beroulle, V.; Cambou, B.; Danger, J.L.; Di Natale, G.; Hely, D.; Guilley, S.; Karimi, N. PUF Enrollment and Life Cycle Management: Solutions and Perspectives for the Test Community. In Proceedings of the 2020 IEEE European Test Symposium (ETS), Tallinn, Estonia, 25-29 May 2020; pp. 1-10.

18. Choi, Y.J.; Kang, H.J.; Lee, I.G. Scalable and secure internet of things connectivity. Electronics 2019, 8, 752. [CrossRef]

19. El-Hajj, M.; Fadlallah, A.; Chamoun, M.; Serhrouchni, A. A survey of internet of things (IoT) Authentication schemes. Sensors 2019, 19, 1141. [CrossRef] [PubMed]

20. Ruhrmair, U.; Solter, J. PUF modeling attacks: An introduction and overview. In Proceedings of the 2014 Design, Automation \& Test in Europe Conference \& Exhibition (DATE), Dresden, Germany, 24-28 March 2014.

21. Fang, Y.; Wang, C.; Ma, Q.; Gu, C.; O’Neill, M.; Liu, W. Attacking Arbiter PUFs Using Various Modeling Attack Algorithms: A Comparative Study. In Proceedings of the 2018 IEEE Asia Pacific Conference on Circuits and Systems (APCCAS), Chengdu, China, 26-30 October 2018; pp. 394-397.

22. Khalafalla, M.; Gebotys, C. PUFs Deep Attacks: Enhanced modeling attacks using deep learning techniques to break the security of double arbiter PUFs. In Proceedings of the 2019 Design, Automation \& Test in Europe Conference \& Exhibition (DATE), Florence, Italy, 25-29 March 2019; pp. 204-209.

23. Barbareschi, M.; Bagnasco, P.; Mazzeo, A. Authenticating iot devices with physically unclonable functions models. In Proceedings of the 2015 10th International Conference on P2P, Parallel, Grid, Cloud and Internet Computing (3PGCIC), Krakow, Poland, 4-6 November 2015; pp. 563-567.

24. Aljafar, M.J.; Acken, J.M. Survey on the benefits of using memristors for PUFs. Int. J. Parallel Emergent Distrib. 2021, [CrossRef]

25. Council, N.R. Counterfeit Deterrent Features for the Next-Generation Currency Design; The National Academies Press: Washington, DC, USA, 1993. [CrossRef]

26. Chatterjee, B.; Das, D.; Maity, S.; Sen, S. RF-PUF: Enhancing IoT security through authentication of wireless nodes using in situ machine learning. IEEE Internet Things J. 2018, 6, 388-398. [CrossRef]

27. McGrath, T.; Bagci, I.E.; Wang, Z.M.; Roedig, U.; Young, R. A PUF taxonomy. Appl. Phys. Rev. 2019, 6, 011303. [CrossRef]

28. Idriss, T.; Idriss, H.; Bayoumi, M. A PUF-based paradigm for IoT security. In Proceedings of the 2016 IEEE 3rd World Forum on Internet of Things (WF-IoT), Reston, VA, USA, 12-14 December 2016; pp. 700-705. [CrossRef]

29. Kusters, L.; Willems, F.M.J. Secret-Key Capacity Regions for Multiple Enrollments With an SRAM-PUF. IEEE Trans. Inf. Forensics Secur. 2019, 14, 2276-2287. [CrossRef]

30. Lee, J.W.; Lim, D.; Gassend, B.; Suh, G.E.; Van Dijk, M.; Devadas, S. A technique to build a secret key in integrated circuits for identification and authentication applications. In Proceedings of the 2004 Symposium on VLSI Circuits. Digest of Technical Papers (IEEE Cat. No. 04CH37525), Honolulu, HI, USA, 25 October 2004; pp. 176-179.

31. Yu, M.D.; M'Raïhi, D.; Verbauwhede, I.; Devadas, S. A noise bifurcation architecture for linear additive physical functions. In Proceedings of the 2014 IEEE International Symposium on Hardware-Oriented Security and Trust (HOST), Arlington, VA, USA, 6-7 May 2014; pp. 124-129.

32. Katzenbeisser, S.; Kocabaş, Ü.; Van Der Leest, V.; Sadeghi, A.R.; Schrijen, G.J.; Wachsmann, C. Recyclable pufs: Logically reconfigurable pufs. J. Cryptogr. Eng. 2011, 1, 177. [CrossRef]

33. Liu, W.; Zhang, L.; Zhang, Z.; Gu, C.; Wang, C.; O'neill, M.; Lombardi, F. XOR-based low-cost reconfigurable PUFs for IoT security. ACM Trans. Embed. Comput. Syst. (TECS) 2019, 18,1-21. [CrossRef]

34. Riedmiller, M.; Braun, H. A direct adaptive method for faster backpropagation learning: The RPROP algorithm. In Proceedings of the IEEE International Conference on Neural Networks, 25-29 October 1993; pp. 586-591.

35. Rührmair, U.; Sölter, J.; Sehnke, F.; Xu, X.; Mahmoud, A.; Stoyanova, V.; Dror, G.; Schmidhuber, J.; Burleson, W.; Devadas, S. PUF modeling attacks on simulated and silicon data. IEEE Trans. Inf. Forensics Secur. 2013, 8, 1876-1891. [CrossRef]

36. Boser, B.E.; Guyon, I.M.; Vapnik, V.N. A training algorithm for optimal margin classifiers. In Proceedings of the Fifth Annual Workshop on Computational Learning Theory, Pittsburgh, PA, USA, 27-29 July 1992; pp. 144-152.

37. Sölter, J. Cryptanalysis of Electrical PUFs Via Machine Learning Algorithms. Available online: https://www.researchgate.net/ profile/Jan-Soelter/publication/259580784_Cryptanalysis_of_electrical_PUFs_via_machine_learning_algorithms/links/00b4 952cc03621836c000000/Cryptanalysis-of-electrical-PUFs-via-machine-learning-algorithms.pdf (accessed on 12 December 2021).

38. Kumar, S.; Niamat, M. Machine learning based Modeling Attacks on a Configurable PUF. In Proceedings of the NAECON 2018-IEEE National Aerospace and Electronics Conference, Dayton, OH, USA, 2018; pp. 169-173.

39. Wang, S.C. Artificial neural network. In Interdisciplinary Computing in Java Programming; Springer: Berlin/Heidelberg, Germany, 2003; pp. 81-100.

40. Suh, G.E.; Devadas, S. Physical unclonable functions for device authentication and secret key generation. In Proceedings of the 2007 44th ACM/IEEE Design Automation Conference, San Diego, CA, USA, 4-8 June 2007; pp. 9-14.

41. Gu, C.; Chang, C.H.; Liu, W.; Yu, S.; Ma, Q.; O'neill, M. A Modeling Attack Resistant Deception Technique for Securing PUF based Authentication. In Proceedings of the 2019 Asian Hardware Oriented Security and Trust Symposium (AsianHOST), Xi'an, China, 16-17 December 2019; pp. 1-6. 
42. Santikellur, P.; Bhattacharyay, A.; Chakraborty, R.S. Deep Learning Based Model Building Attacks on Arbiter PUF Compositions. Technical report, Cryptology ePrint Archive, Report 2019/566. 2019. Available online: https://eprint.iacr.org/2019/566.pdf (accessd on 12 December 2021).

43. KhalafAlla, M. Comprehensive Study of Physical Unclonable Functions on FPGAs: Correlation Driven Implementation, Deep Learning Modeling Attacks, and Countermeasures. 2020. Available online: http://hdl.handle.net/10012/15989 (accessed on 12 December 2021).

44. Hansen, N., The CMA Evolution Strategy: A Comparing Review. In Towards a New Evolutionary Computation: Advances in the Estimation of Distribution Algorithms; Lozano, J.A., Larrañaga, P., Inza, I., Bengoetxea, E., Eds.; Springer: Berlin/Heidelberg, Germany, 2006; pp. 75-102. [CrossRef]

45. Becker, G.T. The Gap between Promise and Reality: On the Insecurity of XOR Arbiter PUFs; International Workshop on Cryptographic Hardware and Embedded Systems; Springer: Berlin/Heidelberg, Germany, 2015; pp. 535-555.

46. Rostami, M.; Majzoobi, M.; Koushanfar, F.; Wallach, D.S.; Devadas, S. Robust and reverse-engineering resilient PUF authentication and key-exchange by substring matching. IEEE Trans. Emerg. Top. Comput. 2014, 2, 37-49. [CrossRef]

47. Kroeger, T.; Cheng, W.; Guilley, S.; Danger, J.L.; Karimi, N. Effect of aging on PUF modeling attacks based on power side-channel observations. In Proceedings of the 2020 Design, Automation \& Test in Europe Conference \& Exhibition (DATE), Grenoble, France, 9-13 March 2020; pp. 454-459.

48. Rabcan, J.; Levashenko, V.; Zaitseva, E.; Kvassay, M.; Subbotin, S. Application of fuzzy decision tree for signal classification. IEEE Trans. Ind. Inf. 2019, 15, 5425-5434. [CrossRef]

49. Kannimuthu, P.; Thangamuthu, J. Decision Tree Trust (DTTrust)-Based Authentication Mechanism to Secure RPL Routing Protocol on Internet of Battlefield Thing (IoBT). Int. J. Bus. Data Commun. Netw. (IJBDCN) 2021, 17, 1-23. [CrossRef]

50. Zimmer, V.; Krau, M. Establishing the Root of Trust. 2016.Available online: https://uefi.org/sites/default/files/resources/ UEFI\%20RoT\%20white\%20paper_Final\%208\%208\%2016\%20(003).pdf (accessed on 12 December 2021).

51. Maes, R. Physically Unclonable Functions: Properties. In Physically Unclonable Functions; Springer: Berlin/Heidelberg, Germany, 2013; pp. 49-80.

52. Cam-Winget, N.; Sadeghi, A.R.; Jin, Y. Can IoT be secured: Emerging challenges in connecting the unconnected. In Proceedings of the 2016 53nd ACM/EDAC/IEEE Design Automation Conference (DAC), Austin, TX, USA, 5-9 June 2016; pp. 1-6.

53. Ammar, M.; Washha, M.; Crispo, B. WISE: Lightweight intelligent swarm attestation scheme for IoT (the verifier's perspective). In Proceedings of the 2018 14th International Conference on Wireless and Mobile Computing, Networking and Communications (WiMob), Limassol, Cyprus, 15-17 October 2018; pp. 1-8.

54. Majzoobi, M.; Elnably, A.; Koushanfar, F. FPGA time-bounded unclonable authentication. In International Workshop on Information Hiding; Springer: Berlin/Heidelberg, Germany, 2010; pp. 1-16.

55. Majzoobi, M.; Koushanfar, F. Time-bounded authentication of FPGAs. IEEE Trans. Inf. Forensics Secur. 2011, 6, 1123-1135. [CrossRef]

56. Hancke, G.P.; Kuhn, M.G. Attacks on Time-of-Flight Distance Bounding Channels. In Proceedings of the First ACM Conference on Wireless Network Security, Alexandria, VA, USA, 31 March-2 April 2008; Association for Computing Machinery: New York, NY, USA, 2008; pp. 194-202. [CrossRef]

57. Brelurut, A.; Gerault, D.; Lafourcade, P. Survey of distance bounding protocols and threats. In International Symposium on Foundations and Practice of Security; Springer: Berlin/Heidelberg, Germany, 2015; pp. $29-49$.

58. Benesty, J.; Chen, J.; Huang, Y.; Cohen, I. Pearson correlation coefficient. In Noise Reduction in Speech Processing; Springer: Berlin/Heidelberg, Germany, 2009; pp. 1-4.

59. Tobisch, J.; Becker, G.T. On the Scaling of Machine Learning Attacks on PUFs with Application to Noise Bifurcation; International Workshop on Radio Frequency Identification: Security and Privacy Issues; Springer: Berlin/Heidelberg, Germany, 2015; pp. 17-31.

60. Gao, Y.; Li, G.; Ma, H.; Al-Sarawi, S.F.; Kavehei, O.; Abbott, D.; Ranasinghe, D.C. Obfuscated challenge-response: A secure lightweight authentication mechanism for PUF-based pervasive devices. In Proceedings of the 2016 IEEE International Conference on Pervasive Computing and Communication Workshops (PerCom Workshops), Sydney, Australia, 14-18 March 2016; pp. 1-6.

61. Delvaux, J. Machine-learning attacks on polypufs, ob-pufs, rpufs, lhs-pufs, and puf-fsms. IEEE Trans. Inf. Forensics Secur. 2019, 14, 2043-2058. [CrossRef]

62. Graf, T.M.; Lemire, D. Xor Filters: Faster and Smaller Than Bloom and Cuckoo Filters. J. Exp. Algorithmics 2019, 25, 2020. [CrossRef]

63. Yilmaz, Y.; Gunn, S.R.; Halak, B. Lightweight PUF-based authentication protocol for IoT devices. In Proceedings of the 2018 IEEE 3rd International Verification and Security Workshop (IVSW), Costa Brava, Spain, 2-4 July 2018; pp. 38-43.

64. Rivest, R.L. The RC5 Encryption Algorithm; International Workshop on Fast Software Encryption; Springer: Berlin/Heidelberg, Germany, 1994; pp. 86-96.

65. Handschuh, H.; Heys, H.M. A Timing Attack on RC5; International Workshop on Selected Areas in Cryptography; Springer: Berlin/Heidelberg, Germany, 1998; pp. 306-318.

66. Miyaji, A.; Nonaka, M.; Takii, Y. Known Plaintext Correlation Attack against RC5; Cryptographers' Track at the RSA Conference; Springer: Berlin/Heidelberg, Germany, 2002; pp. 131-148.

67. Zhang, J.; Shen, C. Set-Based Obfuscation for Strong PUFs Against Machine Learning Attacks. IEEE Trans. Circuits Syst. I Regul. Pap. 2021, 68, 288-300. [CrossRef] 
68. Majzoobi, M.; Koushanfar, F.; Potkonjak, M. Techniques for design and implementation of secure reconfigurable PUFs. ACM Trans. Reconfigurable Technol. Syst. (TRETS) 2009, 2, 1-33. [CrossRef]

69. Adi, Y.; Baum, C.; Cisse, M.; Pinkas, B.; Keshet, J. Turning your weakness into a strength: Watermarking deep neural networks by backdooring. In Proceedings of the 27th USENIX Security Symposium (USENIX Security 18), Baltimore, MD, USA, 15-17 August 2018; pp. 1615-1631.

70. Zhang, J.; Gu, Z.; Jang, J.; Wu, H.; Stoecklin, M.P.; Huang, H.; Molloy, I. Protecting intellectual property of deep neural networks with watermarking. In Proceedings of the 2018 on Asia Conference on Computer and Communications Security, Incheon, Korea, 4-8 June 2018; pp. 159-172. 